\title{
Gasterópodos terrestres de la provincia de Misiones, Argentina
}

\author{
Diego Eduardo Gutiérrez Gregoric, Verónica Núñez, Roberto Eugenio Vogler, \\ Ariel Aníbal Beltramino \& Alejandra Rumi \\ División Zoología Invertebrados, Museo de La Plata, Facultad de Ciencias Naturales y Museo, Universidad Nacional \\ de La Plata, Paseo del Bosque s/nº,B1900WFA, La Plata, Argentina; dieguty@fcnym.unlp.edu.ar,nmariaveronik@ \\ yahoo.com.ar, robertovogler@fcnym.unlp.edu.ar, aabeltramino@fcnym.unlp.edu.ar, alerumi@fcnym.unlp.edu.ar
}

Recibido 21-XI-2012. Corregido 21-IV-2013. Aceptado 27-V-2013.

\begin{abstract}
Terrestrial gastropods from Misiones Province, Argentina. The Atlantic Forest represents one of the global priority eco-regions for biodiversity conservation. In Argentina, this is represented by the Paranense Forest, which occupies much of the Misiones Province. In this paper, an updated list of land mollusks from Misiones Province is presented, and its species richness was evaluated comparing protected and unprotected areas. For this, we reviewed La Plata Museum Mollusk Collection, updated the literature review, and developed some field work between 2009 and 2010. For the areographic work, a grid (50x50km) was applied to a Misiones province map. In this region, large areas of native vegetation constituting the Paranaense Forest still persist, and include protected areas such as the Iguazú National Park and Puerto Península Provincial Park. These areas have been the most explored at the malacological level (both terrestrial and aquatic environments), a fact that is consistent with the greater number of records found, which may also be the explanation for the highest number of species recorded in the region. A database containing 497 records was compiled. The number of species occurring in this Province was increased from 28 to 56; 11 of which were exotic species. A total of 38 of the species occurred in protected areas and 53 in unprotected areas; and 25 species were micro-mollusks. Orthalicidae was the family with the highest species number (10) and the widest distribution, occupying 16/20 sampling units. Megalobulimus was the genus found in a greater number of sampling units (14/20). The highest values of specific richness of land gastropods were found in Northern Misiones $(R=32)$. This richness was similar to that previously reported for the Tucumán Province, although species occurring in both provinces were mostly different. The richness in Misiones Province border areas, as the Republic of Paraguay and the state of Santa Catarina (Brazil), was about twice that found in the province. The present study showed the existence of poorly explored areas at malacological level, especially in Central and Eastern Misiones. Data presented here will allow focus future sampling efforts on such areas, so patterns of species distribution can be better defined. Rev. Biol. Trop. 61 (4): 1759-1768. Epub 2013 December 01.
\end{abstract}

Key words: Gastropoda, distribution, protected areas, exotic species, richness.

El Bosque Atlántico de Sudamérica es una de las eco-regiones considerada prioritaria para la conservación global de la biodiversidad y una de sus veinticinco "zonas calientes de biodiversidad" (biodiversity hotspot; Mittermeier, Myers, Thomsen, Da Fonseca \& Olivieri, 1998, Myers, Mittermeier, Mittermier, Da Fonseca \& Kent, 2000). Este bosque recorre la costa atlántica de Brasil y se extiende hacia el oeste por Paraguay oriental y el noreste de Argentina. En este último país se encuentra representado por la Selva Paranense, la cual ocupa gran parte de la provincia de Misiones. Esta selva posee los remanentes boscosos más grandes del Bosque Atlántico, y éstos todavía contienen el conjunto original de grandes vertebrados (Placci \& Di Bitetti, 2006). A pesar de que la provincia de Misiones solo representa el $1.1 \%$ de la superficie de la Argentina, su biodiversidad, basada en plantas vasculares y vertebrados, es la mayor del país (Giraudo et al., 2005b). Actualmente, se estima que por la actividad antrópica, la 
selva de esta provincia se redujo en un $42.5 \%$ (Holz \& Placci, 2005). Con el fin de proteger este tipo de ambiente y su fauna, en la provincia de Misiones se han generado, alrededor de 60 áreas protegidas públicas y privadas (Giraudo et al., 2005a).

Los invertebrados, salvo excepciones, no han sido tenidos en cuenta en las estimaciones de riqueza específica y diversidad para la provincia de Misiones. Sin embargo, investigaciones realizadas en los últimos años, empleando cuadrículas como método de división del terreno, permitieron registrar 45 especies de gasterópodos de agua dulce, cuatro de las cuales son endémicas de dicha provincia y dos son exóticas (Rumi et al., 2006). Además, muchas de esas especies son vulnerables por poseer requerimientos ecológicos muy específicos (como habitar ambientes de cascada o rápidos) $\mathrm{y}$, por lo tanto, sus distribuciones son restringidas (Rumi et al., 2006). En cuanto a los bivalvos, se registran en esta provincia 29 especies, de las cuales dos son endémicas y dos exóticas (Rumi, Gutiérrez-Gregoric, Núñez \& Darrigran, 2008). En gasterópodos terrestres, el último listado de especies para la Argentina se presenta en Fernández (1973), donde menciona 238 especies para la Argentina y 28 para la provincia de Misiones, y desde esa fecha no se han realizado actualizaciones para la provincia de Misiones.

Los estudios referidos a la riqueza y diversidad de moluscos terrestres en selvas tropicales y subtropicales son escasos, sin embargo, los pocos que se han realizado han demostrado que la malacofauna terrestre en dichos ambientes puede presentar alta riqueza específica, así como elevada diversidad (Salas-Oroño, Cuezzo \& Romero, 2007). Entre los estudios previos realizados en Sudamérica se pueden mencionar los realizados por Gargominy \& Ripken (1998) en Guyana Francesa, Miquel et al. (2007a) en Brasil, Ramírez et al. (2012) en Perú, y Salas-Oroño et al. (2007) y Miranda \& Cuezzo (2010) en la Argentina. Estos dos últimos trabajos realizados en la Selva Nublada (Yungas) de la provincia de Tucumán.
En el presente trabajo se elaboró una actualización de la lista de los moluscos terrestres para la provincia de Misiones, y se evaluó su riqueza al comparar algunas áreas protegidas y no protegidas.

\section{MATERIALES Y MÉTODOS}

Se realizaron muestreos cualitativos en la primavera de 2009 y otoño de 2010, en áreas protegidas (Parque Nacional Iguazú y Reserva Natural de San Antonio -noviembre de 2009 y marzo de 2010-, Parques Provinciales Urugua-í, Piñalito, Puerto Península, Esmeralda, Moconá, Reserva de la Biosfera Yabotí y Reserva de Vida Silvestre Urugua-í -marzo de 2010-) y no protegidas (de cada localidad $\mathrm{N}=1$, marzo de 2010) de la provincia de Misiones, Argentina (Fig. 1). En cada muestreo se revisaron los microhabitats que podrían ser más adecuados para albergar gasterópodos micromoluscos (conchillas de dimensión inferior o igual a los $5 \mathrm{~mm}$ ) y macromoluscos (conchilla mayor a $5 \mathrm{~mm}$, etapa adulta): debajo de troncos caídos, huecos y zona entre raíces de árboles. Para la recolección de especímenes se adaptó la metodología propuesta por Salas-Oroño et al. (2007), donde se realizó en cada sitio tres parcelas de $1 \mathrm{~m}^{2}$ para la visualización de micromoluscos. Posteriormente, la hojarasca fue tamizada y revisada en laboratorio bajo microscopio estereoscópico Leica MZ6. Además, se revisó la colección malacológica del Museo de La Plata (MLP). Los ejemplares en mal estado de conservación (e.g. conchillas rotas y/o falta de partes blandas) no pudieron ser identificados a nivel específico.

Para la identificación supra-genérica se siguió a Bouchet \& Rocroi (2005). Estos autores, además de Miranda \& Cuezzo (2010), Miquel \& Aguirre (2011) y Ramírez et al. (2012) consideran a Orthalicidae una familia con varias subfamilias como Orthalicinae, Bulimulinae (con las Tribus Odontostomini, Bulimulini y Simpulopsini) y Amphibuliminae. Del mismo modo, consideramos a Strophocheilidae con las subfamilias Strophocheilinae y Megalobuliminae como lo mencionan Bouchet 


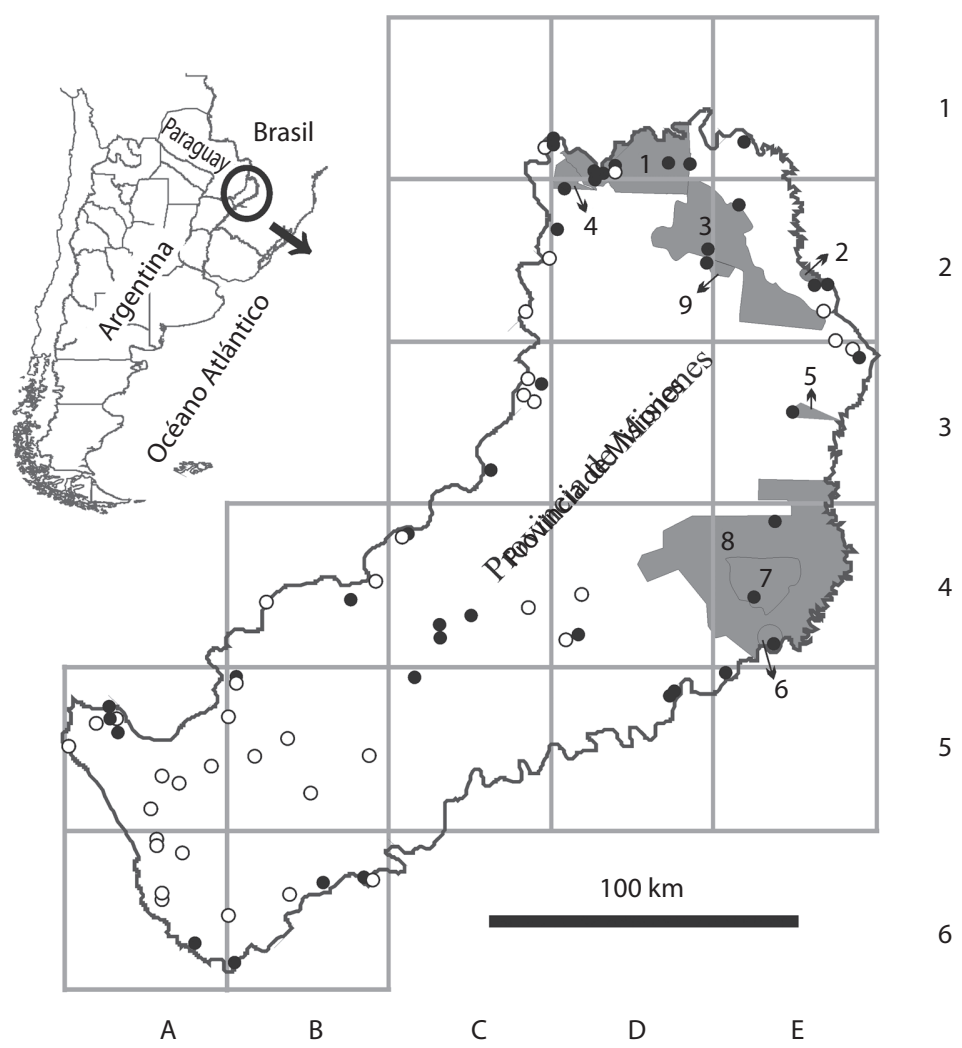

Fig. 1. Provincia de Misiones y áreas protegidas relevadas. 1: Parque Nacional Iguazú; 2: Reserva Natural de San Antonio; 3: Parque Provincial Urugua-í; 4: Parque Provincial Puerto Península; 5: Parque Provincial Piñalito; 6 Parque Provincial Moconá; 7: Parque Provincial Esmeralda; 8: Reserva de la Biosfera Yabotí; 9: Reserva de Vida Silvestre Urugua-í. UM: 50x50km, Letras: columnas; Números: filas. •: Registros de gasterópodos terrestres recolectados en los muestreos cualitativos realizados entre 2009 y 2010; ○: Registros de gasterópodos terrestres depositados en el MLP.

Fig. 1. Misiones Province and relevated protected areas. 1: Iguazú National Park; 2: Natural Reserve of San Antonio; 3: Urugua-í Provincial Park; 4: Puerto Península Provincial Park; 5: Piñalito Provincial Park; 6: Moconá Provincial Park; 7: Esmeralda Provincial Park; 8: Yabotí Biosphere Reserve; 9: Reserve of Vida Silvestre Urugua-í. UM: 50x50km, Letters: columns, Numbers: rows. $\bullet$ : Record of land gastropods collected in qualitative sampling carried out between 2009 and 2010; $\circ$ : Record of land gastropods deposited in MLP.

\& Rocroi (2005), Miquel \& Aguirre (2011) y Ramírez et al. (2012). Para Scolodontidae hemos seguido a Hausdorf (2006), mientras que para Veronicelidae seguimos a Gomes, Britto da Silva, Veitenheimer-Mendes, Thomé \& Bonatto (2010). Como a nivel genérico y específico, no se cuenta con un trabajo actualizado que revise a los gasterópodos terrestres presentes en la Argentina, se siguió a varios autores: Miquel (1989, 1991, 1993, 1995), Campos-Salgado \& Dos Santos-Coelho (2003),
Miquel et al. (2004, 2007a, 2007b), Simone (2006) y Miquel \& Aguirre (2011).

Los registros fueron incorporados a una base de datos, digitalizados y geo-referenciados para luego ser utilizados en sistemas de información geográfica (DIVA-GIS). Para estimar la densidad de registros y riqueza en la provincia, se siguió la metodología empleada por Rabinovich \& Rapoport (1975), Rumi et al. (2006) y Pérez, Sotelo, Arana \& López (2008), utilizando cuadrantes de 50 x $50 \mathrm{~km}$, donde 
cada cuadrante corresponde a una unidad de muestreo (UM, n=20; Fig. 1). Se contabilizó el número de registros por UM. Para calcular la ocupación por géneros y/o familias fueron considerados también aquellos registros en los cuales el material no logró identificarse a nivel específico. Para el análisis de riqueza (R) se consideró el número de especies presentes por UM. En los casos de registrar en una misma UM especies identificadas y no identificadas del mismo género, estas últimas no fueron tenidas en cuenta para el cálculo de riqueza específica por UM.

\section{RESULTADOS}

La exploración cualitativa permitió obtener 259 registros nuevos de gasterópodos terrestres, que sumados a la revisión del MLP totalizan 497 registros. Las campañas realizadas permitieron elevar el número de registros en áreas protegidas a 189 (165 recolectados en los muestreos cualitativos) y a 308 en áreas no protegidas (94 recolectados en los muestreos cualitativos) (Fig. 1). De los 497 registros, 214 pertenecen a micromoluscos (111 recolectados durante 2009 y 2010) y 283 a macromoluscos (148 recolectados entre 2009 y 2010).

La riqueza específica en la provincia de Misiones, producto de los 497 registros obtenidos, fue de 56, representada por 22 familias y 39 géneros (Cuadro 1). En áreas no protegidas se registró 53 de estas especies (18 de manera exclusiva) y en áreas protegidas 38 (tres de manera exclusiva; Cuadro 1). De las 56 especies registradas, 25 correspondieron a micromoluscos (Cuadro 1). Se hallaron gasterópodos en 19 de las 20 UM. A nivel de familias, Orthalicidae

\section{CUADRO 1}

Gasterópodos terrestres en áreas no protegidas (NP) y áreas protegidas (P) de Misiones

TABLE 1

Terrestrial gastropods in unprotected areas (NP) and protected areas (P) of Misiones

\begin{tabular}{|c|c|c|c|c|c|}
\hline ENTIDAD & NP & $\mathrm{P}$ & UM & Ex & Mi \\
\hline \multicolumn{6}{|l|}{ ACHATINIDAE } \\
\hline Achatina fulica Bowdich, 1822 & $\mathrm{x}$ & & $\mathrm{C} 1, \mathrm{D} 1$ & $\mathrm{x}$ & \\
\hline \multicolumn{6}{|l|}{ AGRIOLIMACIDAE } \\
\hline Deroceras sp. Rafinesque, 1820 & & & D1 & & \\
\hline D. leave (Müller, 1774) & $\mathrm{x}$ & $\mathrm{x}$ & A6, C4, D1, E2 & $\mathrm{x}$ & \\
\hline D. reticulatum (Müller, 1774) & $\mathrm{x}$ & $\mathrm{x}$ & $\mathrm{C} 3, \mathrm{D} 1$ & $\mathrm{x}$ & \\
\hline \multicolumn{6}{|l|}{ BRADYBAENIDAE } \\
\hline Bradybaena similaris (Fërussac, 1821) & $\mathrm{x}$ & $\mathrm{x}$ & $\mathrm{A} 5, \mathrm{~B} 5, \mathrm{~B} 6, \mathrm{C} 1, \mathrm{C} 3, \mathrm{C} 4, \mathrm{D} 2, \mathrm{D} 5, \mathrm{E} 1, \mathrm{E} 2$ & $\mathrm{x}$ & \\
\hline \multicolumn{6}{|l|}{ CHAROPIDAE } \\
\hline Lilloiconcha clara (Thiele, 1927) & $\mathrm{x}$ & & B5, D5 & & $\mathrm{x}$ \\
\hline L. gordurasensis (Thiele, 1927) & $\mathrm{x}$ & $\mathrm{x}$ & A6, B5, B6, D1, D5, E3 & & $\mathrm{x}$ \\
\hline L. superba (Thiele, 1927) & $\mathrm{x}$ & & $\mathrm{E} 2, \mathrm{E} 3$ & & $\mathrm{x}$ \\
\hline Radiodiscus compactus (Suter, 1900) & $\mathrm{x}$ & $\mathrm{x}$ & B6, D1, D2, D4, E2, E3 & & $\mathrm{x}$ \\
\hline Rotadiscus amancaezensis (Hidalgo, 1869) & $\mathrm{x}$ & & B5 & & $\mathrm{x}$ \\
\hline \multicolumn{6}{|l|}{ DIPLOMMATINIDAE } \\
\hline Adelopoma paraguayana Parodiz, 1944 & $\mathrm{x}$ & $\mathrm{x}$ & $\mathrm{C} 4, \mathrm{D} 4, \mathrm{E} 3$ & & $\mathrm{x}$ \\
\hline \multicolumn{6}{|l|}{ EUCONULIDAE } \\
\hline Guppya sp. Morch, 1867 & & & D1, D2 & & \\
\hline G. aenea Hylton Scott, 1948 & $\mathrm{x}$ & $\mathrm{x}$ & $\mathrm{A} 5, \mathrm{~B} 5, \mathrm{~B} 6, \mathrm{C} 4, \mathrm{C} 5, \mathrm{D} 1, \mathrm{D} 2, \mathrm{D} 4, \mathrm{D} 5, \mathrm{E} 2, \mathrm{E} 3$ & & $\mathrm{x}$ \\
\hline G. carinata Hylton Scott, 1981 & $\mathrm{x}$ & $\mathrm{x}$ & $\mathrm{B} 5, \mathrm{~B} 6, \mathrm{C} 4, \mathrm{D} 2, \mathrm{E} 2$ & & $\mathrm{x}$ \\
\hline G. disconforme Hylton Scott, 1981 & $\mathrm{x}$ & $\mathrm{x}$ & $\mathrm{C} 4, \mathrm{D} 1$ & & $\mathrm{x}$ \\
\hline Habroconus sp. Fischer, 1872 & & & $\mathrm{~B} 5, \mathrm{D} 1, \mathrm{D} 2, \mathrm{D} 4$ & & \\
\hline
\end{tabular}


CUADRO 1 (Continuación) / TABLE 1 (Continued)

\begin{tabular}{|c|c|c|c|c|c|}
\hline ENTIDAD & NP & $\mathrm{P}$ & UM & Ex & Mi \\
\hline H. lilloana (Hylton Scott, 1948) & $\mathrm{x}$ & $\mathrm{x}$ & $\mathrm{A} 5, \mathrm{~B} 5, \mathrm{~B} 6, \mathrm{C} 4, \mathrm{D} 1, \mathrm{E} 2, \mathrm{E} 3$ & & $\mathrm{x}$ \\
\hline H. semenlini (Moricand, 1845) & $\mathrm{x}$ & $\mathrm{x}$ & $\mathrm{A} 5, \mathrm{~A} 6, \mathrm{~B} 6, \mathrm{C} 4, \mathrm{D} 1, \mathrm{D} 2, \mathrm{E} 2, \mathrm{E} 3, \mathrm{E} 5$ & & $\mathrm{x}$ \\
\hline \multicolumn{6}{|l|}{ FERUSSACIIDAE } \\
\hline Caecilioides consobrina (Orbigny, 1835) & $\mathrm{x}$ & $\mathrm{x}$ & A6, D1, D2 & & $\mathrm{x}$ \\
\hline \multicolumn{6}{|l|}{ GASTRODONTIDAE } \\
\hline Zonitoides arboreus (Say, 1816) & $\mathrm{x}$ & & $\mathrm{A} 5, \mathrm{~B} 5, \mathrm{C} 4, \mathrm{E} 2, \mathrm{E} 3$ & $\mathrm{x}$ & $\mathrm{x}$ \\
\hline \multicolumn{6}{|l|}{ HELICIDAE } \\
\hline Otala sp. Schumacher, 1817 & $\mathrm{x}$ & & B6 & $\mathrm{x}$ & \\
\hline \multicolumn{6}{|l|}{ HELICINIDAE } \\
\hline Helicina sp. Lamarck, 1799 & & & $\mathrm{~B} 5, \mathrm{C} 3, \mathrm{C} 4, \mathrm{D} 2$ & & \\
\hline H. hispida Hylton Scott, 1970 & $\mathrm{x}$ & & $\mathrm{C} 4$ & & $\mathrm{x}$ \\
\hline H. carinata Orbigny, 1835 & $\mathrm{x}$ & $\mathrm{x}$ & $\mathrm{B} 5, \mathrm{~B} 6, \mathrm{C} 3, \mathrm{D} 1$ & & $\mathrm{x}$ \\
\hline H. klappenbachi Weyrauch, 1966 & $\mathrm{x}$ & & D5 & & $\mathrm{x}$ \\
\hline \multicolumn{6}{|l|}{ ORTHALICIDAE } \\
\hline Bulimulus sp. Leach, 1814 & & & $\mathrm{~B} 5, \mathrm{C} 3, \mathrm{C} 4, \mathrm{E} 2, \mathrm{E} 5$ & & \\
\hline B. bonariensis (Rafinesque, 1833) & $\mathrm{x}$ & $\mathrm{x}$ & A5, B6, D1, D2, D5, E2, E3 & & \\
\hline B. fourmiersi Orbigny, 1837 & & $\mathrm{x}$ & D1 & & \\
\hline Cyclodontina sp. Doering, 1874 & & & $\mathrm{C} 4, \mathrm{D} 2, \mathrm{E} 2$ & & \\
\hline C. guarani Orbigny, 1835 & $\mathrm{x}$ & $\mathrm{x}$ & $\mathrm{A} 5, \mathrm{~A} 6, \mathrm{~B} 5, \mathrm{C} 1, \mathrm{C} 3, \mathrm{D} 1, \mathrm{D} 2$ & & \\
\hline Drymaeus sp. Albers, 1850 & & & B4, D1 & & \\
\hline D. interpunctus (Martens, 1866) & $\mathrm{x}$ & $\mathrm{x}$ & $\mathrm{A} 5, \mathrm{~B} 5, \mathrm{C} 1, \mathrm{C} 3, \mathrm{C} 4, \mathrm{D} 1, \mathrm{D} 2$ & & \\
\hline D. papyraceus (Marve, 1823) & $\mathrm{x}$ & $\mathrm{x}$ & $\mathrm{A} 5, \mathrm{~B} 6, \mathrm{C} 3, \mathrm{D} 1$ & & \\
\hline Macrodontes gargantua (Fërussac, 1821) & $\mathrm{x}$ & $\mathrm{x}$ & $\mathrm{A} 5, \mathrm{~B} 5, \mathrm{C} 4, \mathrm{D} 1$ & & \\
\hline Simpulopsis eudioptus (Pilsbry, 1897) & $\mathrm{x}$ & $\mathrm{x}$ & $\mathrm{A} 5, \mathrm{~B} 5, \mathrm{~B} 6, \mathrm{C} 1, \mathrm{C} 2, \mathrm{C} 3, \mathrm{C} 4, \mathrm{D} 1, \mathrm{E} 1, \mathrm{E} 2$, & & \\
\hline S. citrinovitrea (Moricand, 1836) & $\mathrm{x}$ & & B5 & & \\
\hline S. willineri Hylton Scott, 1957 & $\mathrm{x}$ & $\mathrm{x}$ & $\mathrm{B} 4, \mathrm{~B} 5, \mathrm{C} 4, \mathrm{D} 1, \mathrm{D} 2$ & & \\
\hline Spixia sp. Pilsbry \& Vanata, 1898 & & & $\mathrm{C} 4$ & & \\
\hline S. spixii major (d'Orbigny, 1835) & $\mathrm{x}$ & & A5 & & \\
\hline \multicolumn{6}{|l|}{ PHILOMYCIDAE } \\
\hline Meghimatium sp. van Hasselt, 1823 & $\mathrm{x}$ & $\mathrm{x}$ & $\mathrm{B} 4, \mathrm{C} 1, \mathrm{D} 1$ & $\mathrm{x}$ & \\
\hline \multicolumn{6}{|l|}{ PUNCTIDAE } \\
\hline Paralaoma servilis (Shuttleworth, 1852) & $\mathrm{x}$ & & B5 & $\mathrm{x}$ & $\mathrm{x}$ \\
\hline \multicolumn{6}{|l|}{ SCOLODONTIDAE } \\
\hline Happia sp. Bourguignat, 1889 & & & $\mathrm{~A} 5, \mathrm{~A} 6, \mathrm{C} 3, \mathrm{D} 1, \mathrm{D} 2, \mathrm{D} 4, \mathrm{D} 5, \mathrm{E} 2, \mathrm{E} 3$ & & \\
\hline H. insularis (Boettger, 1889) & $\mathrm{x}$ & $\mathrm{x}$ & A5 & & $\mathrm{x}$ \\
\hline Miradiscops brasiliensis (Thiele, 1927) & $\mathrm{x}$ & $\mathrm{x}$ & $\mathrm{A} 5, \mathrm{~A} 6, \mathrm{~B} 6, \mathrm{D} 1, \mathrm{D} 2, \mathrm{D} 4, \mathrm{E} 2, \mathrm{E} 3$ & & $\mathrm{x}$ \\
\hline Scolodonta sp. Doering, 1875 & & & $\mathrm{~A} 5, \mathrm{~A} 6, \mathrm{~B} 5, \mathrm{C} 3, \mathrm{D} 5$ & & \\
\hline S. semperi (Doering, 1875) & $\mathrm{x}$ & & A6, C1, E5 & & $\mathrm{x}$ \\
\hline \multicolumn{6}{|l|}{ SOLAROPSIDAE } \\
\hline Solaropsis heliaca (Orbigny, 1835) & & $\mathrm{x}$ & D1 & & \\
\hline \multicolumn{6}{|l|}{ STREPTAXIDAE } \\
\hline Rectartemon sp. Baker, 1925 & & & $\mathrm{C} 1, \mathrm{C} 3, \mathrm{D} 1, \mathrm{D} 2, \mathrm{D} 5$ & & \\
\hline R. depressus (Hyneman, 1868) & $\mathrm{x}$ & $\mathrm{x}$ & $\mathrm{A} 5, \mathrm{~B} 5, \mathrm{C} 3$ & & \\
\hline R. regius (Löbbecke, 1881) & $\mathrm{x}$ & & A5 & & \\
\hline R. hylephilus (Orbigny, 1835) & $\mathrm{x}$ & $\mathrm{x}$ & $\mathrm{C} 3$ & & \\
\hline Streptaxis sp. Gray, 1837 & $\mathrm{x}$ & $\mathrm{x}$ & $\mathrm{C} 3, \mathrm{D} 1$ & & \\
\hline \multicolumn{6}{|l|}{ STROPHOCHEILIDAE } \\
\hline Anthinus albolabiatus (Jaeckel, 1927) & $\mathrm{x}$ & & A6, B6 & & \\
\hline
\end{tabular}


CUADRO 1 (Continuación) / TABLE 1 (Continued)

\begin{tabular}{|c|c|c|c|c|c|}
\hline ENTIDAD & NP & $\mathrm{P}$ & UM & Ex & Mi \\
\hline Megalobulimus sp. Miller, 1878 & & & $\begin{array}{l}\text { A5, A6, B5, B6, C2, C3, C4, D1, D2, D4, } \\
\text { E2, E3, E4 }\end{array}$ & & \\
\hline M. oblongus (Müller, 1774) & $\mathrm{x}$ & $\mathrm{x}$ & $\mathrm{B} 5, \mathrm{~B} 6, \mathrm{C} 2, \mathrm{C} 4$ & & \\
\hline M. sanctipauli (Ihering \& Pilsbry, 1900) & $\mathrm{x}$ & $\mathrm{x}$ & $\mathrm{C} 1, \mathrm{C} 3, \mathrm{C} 4, \mathrm{D} 1, \mathrm{E} 3, \mathrm{E} 4$ & & \\
\hline \multicolumn{6}{|l|}{ SUBULINIDAE } \\
\hline Subulindae indeterminado & & & B6 & & $\mathrm{x}$ \\
\hline Lamellaxis sp. Strebel \& Pfeiffer, 1882 & $\mathrm{x}$ & & B5 & $\mathrm{x}$ & $\mathrm{x}$ \\
\hline Leptinaria sp. Beck, 1837 & $\mathrm{x}$ & & A5, D5 & & $\mathrm{x}$ \\
\hline Opeas sp. Albers, 1850 & & & A5, B5, B6 & & \\
\hline O. pumilum (Pfeiffer, 1840). & $\mathrm{x}$ & $\mathrm{x}$ & E2 & $\mathrm{x}$ & $\mathrm{x}$ \\
\hline \multicolumn{6}{|l|}{ SUCCINEIDAE } \\
\hline Succinea sp. Draparnaud, 1801 & $\mathrm{x}$ & $\mathrm{x}$ & $\mathrm{A} 5, \mathrm{~A} 6, \mathrm{~B} 4, \mathrm{~B} 6, \mathrm{C} 4, \mathrm{D} 1, \mathrm{D} 5$ & & \\
\hline Omalonix sp. Orbigny, 1841 & $\mathrm{x}$ & & $\mathrm{A} 5, \mathrm{C} 1, \mathrm{D} 1, \mathrm{D} 2$ & & \\
\hline \multicolumn{6}{|l|}{ VALLONIDAE } \\
\hline Pupisoma sp. Stoliczka, 1873 & $\mathrm{x}$ & $\mathrm{x}$ & B6, C4, D1, D2, D4, E2 & & $\mathrm{x}$ \\
\hline \multicolumn{6}{|l|}{ VERONICELLIDAE } \\
\hline Angustipes difficilis (Colosi, 1922) & $\mathrm{x}$ & $\mathrm{x}$ & A6, D1 & & \\
\hline Belocaulus angustipes (Heynemann, 1885) & $\mathrm{x}$ & & A6, D4 & & \\
\hline Phyllocaulis sp. Colosi, 1922 & & & A6, B5, C1, C4, D1, D2, E3 & & \\
\hline P. soleiformis (Orbigny, 1835) & $\mathrm{x}$ & $\mathrm{x}$ & A6, B5, D1 & & \\
\hline P. tuberculosus (Martens, 1868) & $\mathrm{x}$ & $\mathrm{x}$ & B5, E4 & & \\
\hline P. variegatus (Semper, 1885) & $\mathrm{x}$ & $\mathrm{x}$ & $\mathrm{A} 6, \mathrm{~B} 4, \mathrm{~B} 5, \mathrm{C} 4$ & & \\
\hline \multicolumn{6}{|l|}{ VERTIGINIDAE } \\
\hline Vertigo ovata Say, 1822 & & $\mathrm{x}$ & D1 & $\mathrm{x}$ & $\mathrm{x}$ \\
\hline
\end{tabular}

UM: unidades de muestreo ocupadas (ver Fig 1); Ex: exótica; Mi: micromolusco.

UM: sampling unit occupied (see Fig. 1); Ex: exotic; Mi: micro-mollusk.

fue la que presentó el mayor número de especies $(\mathrm{n}=10)$ y distribución más amplia (16 UM; Cuadro 1). Por otra parte, Megalobulimus sp. Miller, 1878 fue el género que se registró en más UM ( $\mathrm{n}=14)$ (Cuadro 1).
La mayor cantidad de registros de gasterópodos $(\mathrm{n}=107)$ se concentró en el extremo norte de la provincia (i.e. $\mathrm{UM}=\mathrm{D} 1$; Cuadro 2), siendo a su vez el área de mayor riqueza específica $(\mathrm{R}=32)$. En esta $\mathrm{UM}$ se reconoció a 16 de las

CUADRO 2

Riqueza (R) y número de gasterópodos terrestres (N) en Misiones, Argentina, en cada unidad de muestreo

TABLE 2

Richness (R) and number of terrestrial gastropods (N) in Misiones province, Argentina, in each sampling unit

\begin{tabular}{ccccccccccccccccccccc} 
& $\mathrm{C} 1$ & $\mathrm{DI}$ & $\mathrm{E} 1$ & $\mathrm{C} 2$ & $\mathrm{D} 2$ & $\mathrm{E} 2$ & $\mathrm{C} 3$ & $\mathrm{D} 3$ & $\mathrm{E} 3$ & $\mathrm{~B} 4$ & $\mathrm{C} 4$ & $\mathrm{D} 4$ & $\mathrm{E} 4$ & $\mathrm{~A} 5$ & $\mathrm{~B} 5$ & $\mathrm{C} 5$ & $\mathrm{D} 5$ & E5 & A6 & B6 \\
$\mathrm{N}$ & 30 & 107 & 2 & 3 & 42 & 33 & 22 & 0 & 24 & 5 & 57 & 9 & 3 & 41 & 47 & 1 & 20 & 3 & 22 & 24 \\
$\mathrm{R}$ & 11 & 32 & 2 & 2 & 19 & 17 & 14 & 0 & 13 & 5 & 23 & 8 & 2 & 22 & 26 & 1 & 11 & 3 & 15 & 18 \\
\hline
\end{tabular}

Código de UM (Letras: columnas; Números: filas, ver Fig. 1).

UM code, Letters: columns, Numbers: rows (see Fig. 1). 
22 familias y el mayor número de especies perteneció a Orthalicidae $(\mathrm{n}=8)$ (Cuadro 1).

\section{DISCUSIÓN}

Los muestreos realizados, la revisión bibliográfica y de la colección del MLP, permitieron elevar el número de especies citadas por Fernández (1973) de 28 a 56 para la provincia de Misiones. Dicha cantidad de especies es inferior a lo informado para áreas limítrofes como Paraguay ( $\mathrm{n}=97)$ y el estado de Santa Catarina, Brasil ( $\mathrm{n}=137$; Quintana, 1982, Agudo-Padrón, 2008, 2011, 2012), у similar a la registrada para Uruguay $(n=59$; Scarabino, 2003). Al igual que en la provincia de Misiones, en todas estas áreas limítrofes la familia con mayor representatividad fue Orthalicidae, que además sustenta el mayor número de especies en Sudamérica. A pesar de que en la provincia de Misiones se registra la mitad de especies que en Santa Catarina, ambas áreas sólo comparten 27 especies y con Paraguay 25. Dado que ambas áreas son limítrofes a Misiones, al este y oeste respectivamente, es probable que parte de esta diferencia en especies se deba a la falta de muestreos intensivos focalizados en gasterópodos terrestres, sobre todo en la Argentina. En este sentido, es de destacar que en Misiones se registran tres especies de Strophocheilidae, mientras que 16 especies son informadas para el estado de Santa Catarina y nueve para Paraguay (Quintana, 1982, AgudoPadrón, 2008).

Con respecto a otras áreas de la Argentina, es poco lo que se conoce acerca de la diversidad de moluscos terrestres, a excepción de los estudios realizados en la provincia de Tucumán (Salas-Oroño et al., 2007, Miranda \& Cuezzo, 2010). En esta última, y particularmente en el área de las Sierras de San Javier se registran 13 familias, 21 géneros y 32 especies de gasterópodos terrestres (Miranda \& Cuezzo, 2010). Estos valores son similares a los registrados en la UM con mayor riqueza de Misiones (D1), con 16 familias, 27 géneros y 35 especies. Es de destacar que la totalidad de las familias referidas para las Sierras de San Javier (a excepción de Xanthonychidae) se encuentran en la provincia de Misiones. Sin embargo, el número de micromoluscos hallados en la provincia de Tucumán fue relativamente mayor al registrado en la provincia de Misiones (31 vs. 25; Salas-Oroño et al., 2007, Miranda \& Cuezzo, 2010), debido probablemente a la realización de estudios más intensivos en este tipo de malacofauna en Tucumán.

De los 11 taxones exóticos registrados en la provincia de Misiones, diez ya habían sido citados para la Argentina (Miquel et al., 2007a, 2007b, Rumi, Sánchez \& Ferrando, 2010, Gutiérrez-Gregoric, Núñez, Vogler \& Rumi, 2011). Se informa por primera vez para la Argentina al género Meghimatium sp. van Hasselt, 1823, el cual todavía no ha podido ser determinado a nivel específico. Este género es originario del este de Asia. Recientemente, Meghimatium pictum (Stoliczka, 1873) fue citada en el sur de Brasil afectando a cultivos de vid, constituyendo éstos los primeros registros de este género por fuera de su área de origen (Gomes et al., 2011). En función de lo precedente, es probable que la especie presente en esta provincia sea $M$. pictum, aunque esta información debe ser confirmada. Entre las especies exóticas recientemente introducidas en Misiones, la última hallada fue Achatina fulica Bowdich, 1822, en la localidad de Puerto Iguazú, al noroeste de dicha provincia (Gutiérrez-Gregoric et al., 2011). Esta especie se ha hallado en grandes densidades, sobre todo en ambientes domiciliarios. Actualmente entidades gubernamentales están realizando tareas de control sobre esta especie (Gutiérrez-Gregoric, Núñez \& Vogler, 2012), debido, principalmente, a su potencial de invasión en el territorio argentino (Vogler et al. 2013). De nuestro estudio se destaca que la especie exótica con mayor distribución es Bradybaena similaris (Fërussac, 1821), la cual estaba citada solo para una localidad (i.e. San Antonio), producto de una recolección realizada por J. Viana en 1956 (Miquel et al., 2007b). La presencia de la familia exótica Helicidae es confirmada por la existencia de un lote presente en la colección del Museo de La Plata (MLP $n^{\circ} 1$ 657) con dos ejemplares de 
Otala sp. recolectados por J. Gregg en 1946 en la localidad de San Javier, sin embargo, no se la ha registrado desde esa fecha, por lo cual es posible suponer que esta especie no se habría establecido.

El número de registros obtenidos en las recolectas propias y de la colección del MLP en áreas protegidas es muy inferior al de áreas no protegidas (189 vs. 308 registros), razón por la cual el número de entidades ha sido superior en las áreas no protegidas (53 vs. 38). Dentro de las áreas protegidas, solo el Parque Nacional Iguazú posee un gran número de registros (92 registros). En otras áreas protegidas como los Parques provinciales Moconá, Esmeralda, Urugua-í y la reserva de la Biosfera Yabotí solo se poseen registros de las campañas realizadas durante el presente trabajo, por lo que la riqueza de dichas áreas puede considerarse subestimada. En este sentido los valores más altos de riqueza se encontraron en el norte de la provincia de Misiones, sitios donde se observa un alto número de registros de gasterópodos terrestres. Esto es coincidente con los valores obtenidos para gasterópodos de agua dulce (Rumi et al., 2006). Muchas de las recolectas en áreas no protegidas se deben a donaciones de personas no vinculadas con la investigación o de investigadores de otras áreas temáticas, que en general, recolectan gasterópodos superiores a los dos centímetros. Nuevas campañas son necesarias para poder elevar el número de registros en áreas poco muestreadas y así poder determinar patrones de distribución.

\section{AGRADECIMIENTOS}

A Parques Nacionales y Provinciales por su buena disposición para asistirnos durante las campañas. Este estudio fue realizado en el marco del proyecto PN636 de la Facultad de Ciencias Naturales y Museo, UNLP y financiado por CREO (Conservation, Research and Education Opportunities), Consejo Nacional de Investigaciones Científicas y Técnicas (CONICET) y Agencia Nacional de Promoción Científica y Tecnológica (ANPCyT; PICT 2042/08).

\section{RESUMEN}

El Bosque Atlántico es una de las eco-regiones prioritarias para la conservación global de la diversidad biológica. En territorio argentino se encuentra representado por la Selva Paranense que ocupa gran parte de la provincia de Misiones. Aquí se presenta una lista actualizada de los moluscos terrestres de la provincia de Misiones, se evalúa su riqueza y se las compara entre áreas protegidas y no protegidas. Se elaboró una base de datos de 497 registros. Se utilizó una grilla de 50x50km sobre el mapa de la provincia de Misiones. Se amplió el número de especies presentes en esta provincia de 28 a 56 ( 11 exóticas y 25 micromoluscos). En áreas no protegidas se registraron 53 especies y 38 en áreas protegidas. Orthalicidae presentó el mayor número de especies (diez) y la distribución más amplia. Megalobulimus spp. se registró en 14 unidades de muestreo. Los mayores valores de riqueza específica se encontraron en el extremo norte de la provincia $(\mathrm{R}=32)$, donde a su vez se registra la mayor cantidad de muestreos. En esta región se mantienen grandes extensiones de vegetación autóctona que conforman la Selva Paranaense y zonas protegidas como el Parque Nacional Iguazú y el Parque Provincial Puerto Península.

Palabras clave: Gastropoda, distribución, áreas protegidas, especies exóticas, riqueza.

\section{REFERENCIAS}

Agudo-Padrón, A. I. (2008). Listagem sistemática dos moluscos continentais ocorrentes no Estado de Santa Catarina, Brasil. Comunicaciones de la Sociedad Malacológica del Uruguay, 9(91), 147-179.

Agudo-Padrón, A. I. (2011). Exotic molluscs in Santa Catarina's State, Southern Brazil region (Mollusca, Gastropoda et Bivalvia): check list and regional spatial distribution knowledge. Biodiversity Journal, 2(2), 53-58.

Agudo-Padrón, A. I. (2012). Nuevos aportes a la lista sistemática de moluscos continentales ocurrentes en el Estado de Santa Catarina, Brasil. Amici Molluscarum, 20(1), 35-42.

Bouchet, P., \& Rocroi, J. P. (2005). Classification and nomenclator of Gastropod families. Malacologia, 47(1-2), 1-397.

Campos-Salgado, N., \& Dos Santos-Coelho, A. C. (2003). Moluscos terrestres do Brasil (Gastrópodes operculados ou não, exclusive Veronicellidae, Milacidae e Limacidae). Revista de Biología Tropical, 51, 149-189.

Fernández, D. (1973). Catálogo de la malacofauna terrestre Argentina, Monografias, 4. La Plata: Comisión de Investigaciones Científicas. 
Gargominy, O., \& Ripken, T. E. J. (1998). Micro-pulmonates in tropical rainforest litter: a new bio-jewel? In R. Bieler, \& P. M. Nikkelsen (Eds.), Abstract, World Congress of Malacology, Washington, D.C. (p. 116). Chicago: Unitas Malacologica.

Giraudo, A. R., Krauczuk, E. R., Arzamendia, V., \& Povedano, H. (2005a). Análise crítica das áreas protegidas na Mata Atlântica da Argentina. In C. Galindo-Leal, \& I. De Gusmão-Câmara (Eds.), Mata Atlântica, biodiversidade, ameaças e perspectivas (p. 245-261). Belo Horizonte: Fundação SOS Mata Atlântica. Conservação Internacional. Centro de Ciências Aplicadas à Biodiversidade.

Giraudo, A. R., Povedano, H., Belgrano, M. J., Krauczuk, E. R., Pardiñas, U., Miquelarena, A., Ligier, D., Baldo, D., \& Castelino, M. (2005b). Status da biodiversidade da Mata Atlântica de Interior da Argentina. In C. Galindo-Leal, \& I. De Gusmão-Câmara (Eds.), Mata Atlâtica, biodiversidade, ameaças e perspectivas (p. 160-180). Belo Horizonte: Fundação SOS Mata Atlântica. Conservação Internacional. Centro de Ciências Aplicadas à Biodiversidade.

Gomes, S. R., Britto da Silva, F., Veitenheimer-Mendes, I. L., Thomé, J. W., \& Bonatto, S. L. (2010). Molecular phylogeny of the South American land slug Phyllocaulis (Mollusca, Soleolifera, Veronicellidae). Zoologica Scripta, 39, 177-186.

Gomes, S. R., Picanço, J. B., Colley, E., Agudo-Padrón, A. I., Nakano, E., \& Thomé, J. W. (2011). A newly introduced and invasive land slug in Brazil: Meghimatium pictum (Gastropoda, Philomycidae) from China. Proceedings of the Academy of Natural Sciences of Philadelphia, 161, 87-95.

Gutiérrez-Gregoric, D. E., Núñez, V., \& Vogler, R. E. (2012). Un gigante africano invade la Argentina. Ciencia Hoy, 22(129), 39-43.

Gutiérrez-Gregoric, D. E., Núñez, V., Vogler, R. E., \& Rumi, A. (2011). Invasion of the Argentinean Paranense Rainforest by the giant African snail Achatina fulica. American Malacological Bulletin, 29(1-2), 135-137.

Hausdorf, B. (2006). The systematic position of Scolodonta Döring, 1875 and Scolodontidae H. B. Baker, 1935 (Gastropoda: Pulmonata). Zoologischer Anzeiger, 245, 161-165.

Holz, S., \& Placci, G. (2005). Raizes socioeconômicas da perda da biodiversidade em Misiones. In C. Galindo-Leal, \& I. De Gusmão-Câmara (Eds.), Mata Atlântica, biodiversidade, ameaças e perspectivas (p. 207-226). Fundação SOS Mata Atlântica. Conservação Internacional. Centro de Ciências Aplicadas à Biodiversidade. Belo Horizonte. Brazil.

Miquel, S. E. (1989). El género Drymaeus Albers, 1850 (Gastropoda, Stylommatophora, Bulimulidae) en la
República Argentina. Studies on Neotropical Fauna \& Environment, 24(2), 75-86.

Miquel, S. E. (1991). El género Bulimus Leach, 1814, (Mollusca, Gastropoda, Stylommatophora) en la República Argentina. Studies on Neotropical Fauna \& Environment, 26(2), 93-112.

Miquel, S. E. (1993). Las especies del género Bostryx Troschel 1847 en la República Argentina (1ra. parte) (Gastropoda Stylommatophora: Bulimulidae). Archiv für Molluskenkunde, 121: 157-171.

Miquel, S. E. (1995). Las especies del género Bostryx Troschel 1847 en la República Argentina (2da. Parte y última parte) (Gastropoda: Stylommatophora: Bulimulidae). Archiv für Molluskenkunde, 124: 119-127.

Miquel, S. E. \& Aquirre, M. L. (2011). Taxonomía de los gasterópodos terrestres del Cuaternario de Argentina. Revista Española de Paleontología, 26(2), 101-133.

Miquel, S. E., Ramírez, R., \& Thomé, J. W. (2004). Lista preliminar de los Punctoideos de Rio Grande do Sul, Brasil, con descripción de dos especies nuevas (Mollusca, Gastropoda, Stylommatophora). Revista Brasileira de Zoologia, 21(4), 925-935.

Miquel, S. E., Ramírez, R., \& Thomé, J. W. (2007a). Biodiversidad y taxonomía de micromoluscos Punctoidea del sur de Brasil, con la descripción de una nueva especie de Radiodiscus de la Mata Atlántica (Mollusca, Gastropoda, Pulmonata). Revista del Museo Argentino de Ciencias Naturales, 9(2), 205-230.

Miquel, S. E., Tablado, A., \& Sodor, A. (2007b). Curaduría en la Colección Nacional de Invertebrados de Argentina: aportes a la biodiversidad y biogeografía de gasterópodos terrestres argentinos. Comunicaciones de la Sociedad Malacológica del Uruguay, 9(90), 113-115.

Miranda, M. J., \& Cuezzo, M. G. (2010). Biodiversidad de gasterópodos terrestres (Mollusca) en el Parque Biológico Sierra de San Javier, Tucumán, Argentina. Revista de Biología Tropical, 58(3), 1009-1029.

Mittermeier, R. A., Myers, N., Thomsen, J. B., Da Fonseca, G. A. B., \& Olivieri, S. (1998). Biodiversity hotspots and major tropical wilderness areas: approaches to setting conservation priorities. Conservation Biology, 12(3), 516-520.

Myers, N., Mittermeier, R. A., Mittermier, C. G., Da Fonseca, G. A. B., \& Kent, J. (2000). Biodiversity hotspots for conservation priorities. Nature, 403: 853-858.

Quintana, M. G. (1982). Catálago preliminar de la malacofauna del Paraguay. Revista del Museo Argentino de Ciencias Naturales Bernardino Rivadavia 11(3), 61-158.

Pérez, A. M., Sotelo, M., Arana, I., \& López, A. (2008). Diversidad de moluscos gasterópodos terrestres en la región del Pacífico de Nicaragua y sus preferencias 
de hábitat. Revista de Biología Tropical, 56(1), 317-332.

Placci, G., \& Di Bitetti, M. (2006). Situación ambiental en la ecorregión del Bosque Atlántico del Alto Paraná (Selva Paranaense). In A. Brown, U. MartinezOrtiz, M. Acerbi \& J. Corcuera (Eds.), La Situación Ambiental Argentina 2005 (p. 197-210). Buenos Aires: Fundación Vida Silvestre Argentina.

Rabinovich, J. E., \& Rapoport, E. H. (1975). Geographical variation of diversity in Argentina passerine birds. Journal of Biogeography, 2, 141-157.

Ramírez, R., Borda, V., Romero, P., Ramírez, J., Congrains, C., Chirinos, J., Ramírez, P., Velásquez, L. E., \& Mejía, K. (2012). Biodiversidad y endemismo de los caracoles terrestres Megalobulimus y Systrophia en la Amazonia occidental. Revista Peruana de Biología, 19(1), 59-74.

Rumi, A., Gutiérrez-Gregoric, D. E., Núñez, V., César, I. I., Roche, M. A., Tassara, M. P., Martín, S. M., \& López Armengol, M. F. (2006). Freshwater Gastropoda from Argentina: species richness, distribution patterns, and an evaluation of endangered species. Malacologia, 49(1), 189-208.
Rumi, A., Gutiérrez-Gregoric, D. E., Núñez, V., \& Darrigran, G. A. (2008). Malacología Latinoamericana. Moluscos de agua dulce de Argentina. Revista de Biología Tropical, 56(1), 77-111.

Rumi, A., Sánchez, J., \& Ferrando, N. S. (2010). Theba pisana (Müller, 1774) (Gastropoda, Helicidae) and other alien land molluscs species in Argentina. Biological Invasions, 12, 2985-2990.

Salas Oroño, E., Cuezzo, M. G., \& Romero, F. (2007). Land snail diversity in subtropical rainforest mountains (Yungas) of Tucumán, northwestern Argentina. American Malacological Bulletin, 22, 17-26.

Scarabino, F. (2003). Lista sistemática de los Gastropoda terrestres vivientes de Uruguay. Comunicaciones de la Sociedad Malacológica del Uruguay, 8(78-79), 203-214.

Simone, L. R. L. (2006). Land and freshwater molluscs of Brazil. São Paulo: EGB, Fapesp.

Vogler, R. E., Beltramino, A. A., Sede, M. M., Gutiérrez Gregoric, D. E., Núñez, V., \& Rumi, A. (2013). The Giant african snail, Achatina fulica (Gastropoda: Achatinidae): using bioclimatic models to identify South American areas susceptible to invasion. American Malacological Bulletin, 31, 39-50. 